\title{
Robust stabilization of nonlinear systems via stable kernel representations with $L_{2}$-gain bounded uncertainty
}

\author{
A.J. van der Schaft ${ }^{1}$ \\ Systems and Control Group, Department of Applied Mathematics, University of Twente, P.O. Box 217, 7500 \\ AE Enschede, Netherlands
}

(Received 24 November 1993)

\begin{abstract}
The approach to robust stabilization of linear systems using normalized left coprime factorizations with $\mathscr{H}_{\infty}$ bounded uncertainty is generalized to nonlinear systems. A nonlinear perturbation model is derived, based on the concept of a stable kernel representation of nonlinear systems. The robust stabilization problem is then translated into a nonlinear disturbance feedforward $\mathscr{H}_{\infty}$ optimal control problem, whose solution depends on the solvability of a single Hamilton-Jacobi equation.
\end{abstract}

Keywords: Robust nonlinear control; Perturbation model; Kernel representation; Small-gain theorem; Nonlinear $\mathscr{H}_{\infty}$ control

\section{Introduction}

A wealth of literature is available on the problem of robustly stabilizing nonlinear uncertain systems. Here we propose a very particular approach, which directly generalizes the solution of the linear robust stabilization problem via normalized left coprime factorizations, as obtained in Glover and McFarlane [6] (see also [15]), to the nonlinear case. Essential ingredients in our approach are the stable kernel representation of nonlinear state space systems as introduced in $[18,17]$, the resulting nonlinear perturbation model, and the solution to a particular type of nonlinear $\mathscr{H}_{\infty}$ control problems. The theory is illustrated with a simple example admitting an explicit solution.

\section{A nonlinear perturbation model}

A very general perturbation model for linear systems is the numerator-denominator perturbation model, or coprime factor uncertainty model, as it is also known (see e.g. $[23,12,24]$ ). Let $G(s)$ be the transfer matrix of a linear system (i.e. $G(s)$ is a proper rational matrix). Left factorization of $G(s)$ over the stable proper rational

\footnotetext{
${ }^{1}$ Also: CWI, Amsterdam, Netherlands. 
matrices yields $G(s)=D^{-1}(s) N(s)$, with $D(s), N(s)$ coprime stable proper rational matrices. The stable linear system

$$
e=[N(s) \vdots-D(s)]\left[\begin{array}{l}
u \\
y
\end{array}\right]
$$

(with "inputs" $\left[\begin{array}{l}u \\ y\end{array}\right]$ and "outputs" $e$ ) will be called a stable kernel representation of $G(s)$, since by setting $e=0$ in (1) one recovers the input-output map $y=G(s) u$. In the numerator-denominator perturbation model one considers the following class of perturbations:

$$
\begin{aligned}
& N(s) \mapsto N(s)+\Delta_{N}(s), \\
& D(s) \mapsto D(s)+\Delta_{D}(s),
\end{aligned}
$$

with $\Delta_{N}(s), \Delta_{D}(s)$ stable proper rational matrices. (In applications one would normally include some extra weighting filters; however, they can be incorporated in the system transfer matrix $G(s)$, see $[15,13]$.) This results in the perturbed stable kernel representation

$$
e_{\mathrm{p}}=[N(s) \vdots-D(s)]\left[\begin{array}{l}
u \\
y
\end{array}\right]+\left[\Delta_{N}(s) \vdots-\Delta_{D}(s)\right]\left[\begin{array}{l}
u \\
y
\end{array}\right]
$$

and the perturbed transfer matrix $G_{\mathrm{p}}(s)=\left[D(s)+\Delta_{D}(s)\right]^{-1}\left[N(s)+\Delta_{N}(s)\right]$. Usually it is convenient to normalize the kernel representation (1) by starting with left coprime factors $D(s), N(s)$ satisfying

$$
N(s) N^{\mathrm{T}}(-s)+D(s) D^{\mathrm{T}}(-s)=I, \quad s \in \mathbb{C} .
$$

A detailed treatment of the robust stabilization problem based on this normalized coprime factor uncertainty model is given in $[6,15]$, see also [24] for the unnormalized case.

Now let us consider smooth nonlinear systems

$$
\Sigma: \begin{aligned}
& \dot{x}=f(x)+g(x) u, \quad u \in \mathbb{R}^{m}, \\
& y=h(x), \quad y \in \mathbb{R}^{p},
\end{aligned}
$$

where $x=\left(x_{1}, \ldots, x_{n}\right)$ are local coordinates for an $n$-dimensional state space manifold $M$. Throughout we assume the existence of a distinguished equilibrium $x_{0}$, i.e. $f\left(x_{0}\right)=0$. Without loss of generality we assume $x_{0}=0$, and furthermore $h(0)=0$.

Before defining a stable kernel representation for $\Sigma$ and the resulting perturbation model we need some preliminaries. Let $\gamma>0$. $\Sigma$ is said to have $L_{2}$-gain $\leqslant \gamma$ if there exists a nonnegative solution $V: M \rightarrow \mathbb{R}$ (a storage function) to the dissipation inequality [25],

$$
V\left(x\left(t_{1}\right)\right)-V\left(x\left(t_{0}\right)\right) \leqslant \frac{1}{2} \int_{t_{0}}^{t_{1}}\left(\gamma^{2}\|u(t)\|^{2}-\|y(t)\|^{2}\right) \mathrm{d} t, \quad V(0)=0,
$$

for all $t_{1} \geqslant t_{0}$ and all $u \in L_{2}\left[t_{0}, t_{1}\right]$ (with $x\left(t_{1}\right)$ denoting the solution at time $t_{1}$ for initial condition $x\left(t_{0}\right)$ at time $t_{0}$ ). $\Sigma$ is said to have $L_{2}$-gain $<\gamma$ if there exists some $\tilde{\gamma}<\gamma$ such that $\Sigma$ has $L_{2}$-gain $\leqslant \tilde{\gamma}$. Throughout we will assume that if there exists a solution $V \geqslant 0$ to (6) then there also exists a differentiable solution $V \geqslant 0$ to (6), and we will restrict ourselves to these differentiable solutions.

Let $\Sigma$ have $L_{2}$-gain $\leqslant \gamma$. From $[7,8,21]$ we recall that if additionally $\Sigma$ is zero-state observable (i.e. $y(t)=0, u(t)=0, \forall t \geqslant 0$, implying $x(0)=0$ ), then necessarily a solution $V \geqslant 0$ to (6) is positive definite $(V(x)>0, x \neq 0)$, and 0 is a locally asymptotically stable equilibrium of (5) with Lyapunov function $V$.

Next we consider the Hamilton-Jacobi-Bellman equation (corresponding to $\Sigma$ with cost criterion $\left.\int_{-\infty}^{0}\left(\|u(t)\|^{2}+\|y(t)\|^{2}\right) \mathrm{d} t\right)$

$$
W_{x}(x) f(x)+\frac{1}{2} W_{x}(x) g(x) g^{\mathrm{T}}(x) W_{x}^{\mathrm{T}}(x)-\frac{1}{2} h^{\mathrm{T}}(x) h(x)=0, \quad W(0)=0,
$$


where

$$
W_{x}(x)=\left(\frac{\partial W}{\partial x_{1}}(x), \ldots, \frac{\partial W}{\partial x_{n}}(x)\right) .
$$

The following theorem is a generalized version of $[18,17]$.

Theorem 2.1. Consider the nonlinear system (5), and assume it is zero-state detectable $(y(t)=0, u(t)=0$, $\forall t \geqslant 0$, implying $x(t) \rightarrow 0, t \rightarrow \infty)$. Suppose there exists a smooth positive definite solution $W$ to (7), and suppose there exists a smooth solution $k(x)$ to

$$
W_{x}(x) k(x)=h^{\mathrm{T}}(x)
$$

Define the system $\Sigma_{\mathrm{s}}$ with inputs $\left[\begin{array}{l}u \\ y\end{array}\right]$ and outputs $e$ :

$$
\begin{aligned}
\dot{x} & =[f(x)-k(x) h(x)]+[g(x) \vdots k(x)]\left[\begin{array}{l}
u \\
y
\end{array}\right], \\
\Sigma_{\mathrm{s}}: \quad e & =h(x)-y .
\end{aligned}
$$

Then $f(x)-k(x) h(x)$ is locally asymptotically stable (w.r.t. the equilibrium $x=0$ ) with Lyapunov function $W$, and globally asymptotically stable if $W$ is proper (i.e. the sets $\{x \in M \mid 0 \leqslant W(x) \leqslant c\}$ are compact for every $c \geqslant 0$ ). Furthermore, $\Sigma_{\mathrm{s}}$ has $L_{2}$-gain $=1$. Setting $e=0$ in $\Sigma_{\mathrm{s}}$ yields $\Sigma$, and $\Sigma_{\mathrm{s}}$ will be called a (nonlinear) stable kernel representation of $\Sigma$.

Proof (sketch, see $[18,17]$ for details). From (7) and (8) we obtain

$$
W_{x}(x)[f(x)-k(x) h(x)]=-\frac{1}{2} W_{x}(x) g(x) g^{\mathrm{T}}(x) W_{x}^{\mathrm{T}}(x)-\frac{1}{2} h^{\mathrm{T}}(x) h(x) \leqslant 0
$$

and (global) asymptotic stability follows from LaSalle's invariance principle. Similarly,

$$
\begin{aligned}
& W_{x}(x)([f(x)-k(x) h(x)]+g(x) u+k(x) y) \\
& \quad=-\frac{1}{2}\left\|u-g^{\mathrm{T}}(x) W_{x}^{\mathrm{T}}(x)\right\|^{2}-\frac{1}{2}\|e\|^{2}+\frac{1}{2}\|u\|^{2}+\frac{1}{2}\|y\|^{2},
\end{aligned}
$$

proving $L_{2}$-gain $\leqslant 1$ by integration (see e.g. [21]).

Remark 2.2. If the linearized system $\dot{x}=(\partial f / \partial u)(0) x+g(0) u, y=(\partial h / \partial x)(0) x$ is anti-stabilizable, and if the imaginary eigenvalues of $(\partial f / \partial x)(0)$ are $(\partial h / \partial x)(0)$-observable, then at least locally around 0 there exists a smooth non-negative solution $W \geqslant 0$ to (7) (see e.g. $[11,20]$ ), which will be locally positive definite if the linearized system is observable.

Remark 2.3. Consider a star-shaped coordinate neighborhood of $x=0$. Since $W_{x}(0)=0$ and $h(x)=0$ we can write (see e.g. [16])

$$
W_{x}(x)=x^{\mathrm{T}} M(x), \quad h(x)=C(x) x
$$

for suitable matrices $M(x), C(x)$, with entries depending smoothly on $x$. Assume that $M(x)$ is invertible for all $x$ in the coordinate neighborhood of 0 ; then a solution $k(x)$ to $(8)$ is given as [18]

$$
k(x)=M^{-1}(x) C^{\mathbf{T}}(x) .
$$


(See [9] for similar considerations in a different context.) Note furthermore that $M(0)=\left(\partial^{2} W / \partial x^{2}\right)(0)$ (the Hessian matrix of $W$ at 0 ). Thus, under the conditions of Remark 1, $M(0)$ will be positive definite, implying that $M(x)$ will be invertible for $x$ near 0 .

Remark 2.4 ([18]). If $-V$ is a negative definite solution to (7), then

$$
\begin{aligned}
\Sigma_{\mathrm{ri}}: & \dot{p}=f(p)-g(p) g^{\mathrm{T}}(p) V_{\mathrm{p}}^{\mathrm{T}}(p)-k(p) e, \quad p \in M, \\
& {\left[\begin{array}{l}
u \\
y
\end{array}\right]=\left[\begin{array}{l}
-g^{\mathrm{T}}(p) V_{\mathrm{p}}^{\mathrm{T}}(p) \\
h(p)-e
\end{array}\right] }
\end{aligned}
$$

is a right inverse system to $\Sigma_{\mathrm{s}}$, i.e., if $x(0)=p(0)$, then the input-output map of $\Sigma_{\mathrm{s}} \circ \Sigma_{\mathrm{ri}}$ is the identity mapping. Furthermore, $f(p)-g(p) g^{\mathbf{T}}(p) V_{\mathrm{p}}^{\mathrm{T}}(p)$ is locally asymptotically stable (w.r.t. $p=0$ ) with Lyapunov function $V$ (and globally asymptotically stable if $V$ is proper). Hence $\Sigma_{\mathrm{s}}$ has a stable right inverse, generalizing the linear notion of coprimeness.

Remark 2.5. For a linear system $\Sigma$, the stable kernel representation $\Sigma_{\mathrm{s}}$ reduces to the left normalized coprime factorization (1), (4).

Analogously to the linear case (cf. (3)), we will now consider perturbed nonlinear stable kernel representations

$$
\begin{aligned}
& \dot{x}=[f(x)-k(x) h(x)]+[g(x): k(x)]\left[\begin{array}{l}
u \\
y
\end{array}\right], \\
& e_{\mathrm{p}}=e+w
\end{aligned}
$$

where $w$ is the output of an arbitrary nonlinear state space system with input $\left[\begin{array}{l}u \\ y\end{array}\right]$,

$$
\Delta: \begin{array}{ll}
\dot{\varphi}=\alpha(\varphi, u, y), & \alpha(0,0,0)=0 \\
w=\beta(\varphi, u, y), & \beta(0,0,0)=0
\end{array}
$$

having finite $L_{2}$-gain. (More generally we could consider families of nonlinear input-output maps from $\left[\begin{array}{l}u \\ y\end{array}\right]$ to $w$, parametrized by the set of initial conditions.) Setting $e_{\mathrm{p}}=0$ in (15) yields the perturbed system

$$
\Sigma_{\mathbf{p}}: \begin{aligned}
& \dot{x}=f(x)+g(x) u+k(x) w, \\
& y=h(x)+w,
\end{aligned}
$$

with $w$ the output of (16).

\section{The robust stabilization problem}

Consider the nonlinear system $\Sigma$ given by (5), and its perturbed model $\Sigma_{\mathrm{p}}$ given by (17), (16). The robust stabilization problem is to find a controller

$$
C: \quad \begin{aligned}
& \dot{\xi}=l(\xi, y), \quad l(0,0)=0, \\
& u=m(\xi, y), \quad m(0,0)=0,
\end{aligned}
$$

with $\xi \in \mathbb{R}^{c}$ the controller state, such that the $L_{2}$-gain of the closed-loop system (17), (18), from w to $z=\left[\begin{array}{l}u \\ y\end{array}\right]$, is minimized, say equal to $\gamma^{*} \geqslant 0$. 
By the small-gain theorem (see e.g. [3]) this will mean that the overall closed-loop system (16)-(18) will be $L_{2}$-stable for all perturbations $\Delta$ with $L_{2}$-gain strictly less than $1 / \gamma^{*}$.

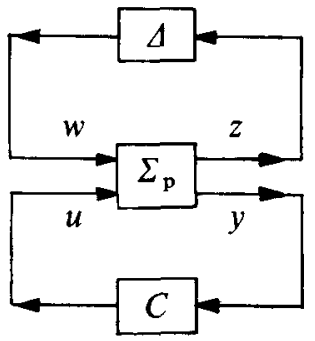

In state space terms, if there exist proper positive definite solutions $V_{\Sigma C}, V_{\Delta}$ to the dissipation inequalities

$$
\begin{aligned}
& V_{\Sigma C}\left(x\left(t_{1}\right), \xi\left(t_{1}\right)\right)-V_{\Sigma C}\left(x\left(t_{0}\right), \xi\left(t_{0}\right)\right) \leqslant \frac{1}{2} \int_{t_{0}}^{t_{1}}\left(\gamma^{2}\|w(t)\|^{2}-\|z(t)\|^{2}\right) \mathrm{d} t, \quad \gamma \geqslant \gamma^{*}, \\
& V_{\Delta}\left(\varphi\left(t_{1}\right)\right)-V_{\Delta}\left(\varphi\left(t_{0}\right)\right) \leqslant \frac{1}{2} \int_{t_{0}}^{t_{1}}\left(\gamma_{\Delta}^{2}\|z(t)\|^{2}-\|w(t)\|^{2}\right) \mathrm{d} t, \quad \gamma_{\Delta}<1 / \gamma,
\end{aligned}
$$

then, assuming zero-state detectability [7], the overall closed-loop system (16)-(18) will be globally asymptotically stable with Lyapunov function $\gamma_{\Delta} V_{\Sigma C}+\gamma V_{\Delta}$, as can be readily checked from (19).

The problem of minimizing the $L_{2}$-gain from $w$ to $z=\left[\begin{array}{l}u \\ y\end{array}\right]$ for (17) is a standard $\mathscr{H}_{\infty}$ optimal nonlinear control problem $[19,21,10,1,22,9]$. Usually one first considers the suboptimal $\mathscr{H}_{\infty}$ problem of finding for given $\gamma>0$ a controller $C$ (if existing!) which makes the $L_{2}$-gain from $w$ to $z=\left[\begin{array}{l}u \\ y\end{array}\right]$ less than or equal to $\gamma$. For the solution to the suboptimal $\mathscr{H}_{\infty}$ control problem we follow the approach of $[2,22]$. For the state feedback suboptimal $\mathscr{H}_{\infty}$ control problem we consider the pseudo-Hamiltonian

$$
\begin{aligned}
K(x, p, u, w)= & p^{\mathrm{T}}[f(x)+g(x) u+k(x) w] \\
& -\frac{1}{2} \gamma^{2}\|w\|^{2}+\frac{1}{2}\|u\|^{2}+\frac{1}{2}\|h(x)+w\|^{2} .
\end{aligned}
$$

Solving $\partial K / \partial u=0, \partial K / \partial d=0$ leads to the saddle point $u^{*}=-g^{\mathrm{T}}(x) p, w^{*}=\left(\gamma^{2}-1\right)^{-1}\left[h(x)+k^{\mathrm{T}}(x) p\right]$. Substitution of $u^{*}, w^{*}$ into $K$ yields the Hamiltonian $H(x, p)=K\left(x, p, u^{*}, w^{*}\right)$ and the HamiltonJacobi-Isaacs equation $H\left(x, P_{x}^{\mathrm{T}}(x)\right)=0$ given as

$$
\begin{aligned}
& P_{x}(x)\left[f(x)+\left(\gamma^{2}-1\right)^{-1} k(x) h(x)\right]+\frac{1}{2} \gamma^{2}\left(\gamma^{2}-1\right)^{-1} h^{\mathrm{T}}(x) h(x) \\
& \quad+\frac{1}{2} P_{x}(x)\left[\left(\gamma^{2}-1\right)^{-1} k(x) k^{\mathrm{T}}(x)-g(x) g^{\mathrm{T}}(x)\right] P_{x}^{\mathrm{T}}(x)=0, \quad P(0)=0 .
\end{aligned}
$$

If there exists a solution $P \geqslant 0$ to (21) then the suboptimal state feedback $\mathscr{H}_{\infty}$ control problem (for $\gamma$ ) is solvable by the state feedback

$$
u=-g^{\mathrm{T}}(x) P_{x}^{\mathrm{T}}(x) \text {. }
$$

Following the certainty equivalence principle of [2] the solution to the output feedback suboptimal $\mathscr{H}_{\infty}$ control problem is, under appropriate conditions, given as

$$
u=-g^{\mathrm{T}}(\hat{x}) P_{x}^{\mathrm{T}}(\hat{x}),
$$

with $\hat{x}(t)$ denoting the worst-case estimate of $x(t)$ given the measurements $y(\tau),-\infty<\tau \leqslant t$, see [2, 22]. (Currently there is intense research activity about the precise conditions for the validity of the worst-case certainty equivalence principle, but we will not elaborate on this.) In general (see e.g. [22]), this will yield an infinite dimensional controller. In the present case, however, the situation is much simpler. Indeed, the suboptimal $\mathscr{H}_{\infty}$ control problem for (17) with $z=\left[\begin{array}{l}u \\ y\end{array}\right]$ is an example of the so-called disturbance feedforward problem, discussed for the linear case in [5], and for the nonlinear case in [14]. In fact, by asymptotic stability 
of $\dot{x}=f(x)-k(x) h(x)$ it follows that for a given control function $u(\tau),-\infty<\tau \leqslant t$, the measurement record $y(\tau),-\infty<\tau \leqslant t$, uniquely specifies the disturbance $\hat{w}(\tau)=y(\tau)-h(\hat{x}(\tau))$ and the state trajectory $\hat{x}(\tau)$. Indeed the state trajectory $\hat{x}(\cdot)$ is generated by the differential equations

$$
\dot{\hat{x}}(\tau)=f(\hat{x}(\tau))+g(\hat{x}(\tau)) u(\tau)+k(\hat{x}(\tau))[y(\tau)-h(\hat{x}(\tau))], \quad x(-\infty)=0 .
$$

Hence a controller solving the suboptimal $\mathscr{H}_{\infty}$ control problem for (17) is given as (substitute (23) into (24))

$$
\begin{aligned}
& \dot{\hat{x}}=f(\hat{x})-g(\hat{x}) g^{\mathrm{T}}(\hat{x}) P_{x}^{\mathrm{T}}(\hat{x})+k(\hat{x})[y-h(\hat{x})], \\
& u=-g^{\mathrm{T}}(\hat{x}) P_{x}^{\mathrm{T}}(\hat{x}) .
\end{aligned}
$$

Based on the linear case, the same controller for the general nonlinear disturbance feedforward problem has been recently proposed in [14]. In this paper also a direct proof is provided showing that (25) solves the suboptimal $\mathscr{H}_{\infty}$ control problem at least locally, i.e. for initial states in a neighborhood of the origin and for disturbances $w(\cdot)$ which keep the state trajectories within this neighborhood. Summarizing, we have the following theorem.

Theorem 3.1. Suppose (cf. Theorem 2.1) that $\Sigma_{\mathrm{s}}$ given by (9) is a stable kernel representation of $\Sigma$ such that $f(x)-k(x) h(x)$ is globally asymptotically stable. Suppose there exists a solution $P \geqslant 0$ to (21) for given $\gamma>0$, and assume the certainty equivalence principle for the suboptimal $\mathscr{H}_{\infty}$ control problem for (17) holds. Then the controller (25) stabilizes the closed-loop system (16), (17), (25) for every perturbation system $\Delta$ as in (16), having $L_{2}$-gain $<1 / \gamma$.

Remark 3.2. From the linear theory (cf. $[6,15]$ ) and the local existence of solutions to (21) based on existence of solutions to the corresponding Riccati equation (cf. $[20,21]$ ), it follows that the minimal $\gamma^{*}$, such that locally around 0 there exist solutions $P \geqslant 0$ to $(21)$ for $\gamma>\gamma^{*}$, is given by

$$
\gamma^{*}=\left[1+\sigma_{\max }(X Z)\right]^{1 / 2},
$$

with $X$ the Hessian matrix $\left(\partial^{2} V / \partial x^{2}\right)(0)$ and $Z$ the inverse Hessian matrix $\left[\left(\partial^{2} W / \partial x^{2}\right)(0)\right]^{-1}$ of the solutions $W \geqslant 0$ and $-V \leqslant 0$ to (7).

Remark 3.3. A related approach to nonlinear robust stabilization will be found in [4].

Example 3.4. Let $\Sigma$ be a lossless system, i.e. there exists $H: M \rightarrow \mathbb{R}, H(0)=0, H(x)>0, x \neq 0$, called the internal energy, such that $(\mathrm{d} / \mathrm{d} t) H=u^{\mathrm{T}} y$ or, equivalently,

$$
H_{x}(x) f(x)=0, \quad H_{x}(x) g(x)=h^{\mathrm{T}}(x) .
$$

Clearly, positive and negative definite solutions to (7) are given as $H$, and $-H$, respectively. Furthermore, $k(x)$ solving (8) is given as $g(x)$, and thus the perturbed system $\Sigma_{\mathbf{p}}$ is given as

$$
\dot{x}=f(x)+g(x)[u+w], \quad y=g^{\mathrm{T}}(x) H_{x}^{\mathrm{T}}(x)+w .
$$

The Hamilton-Jacobi-Isaacs equation (21) takes the form

$$
\begin{aligned}
& P_{x}(x)\left[f(x)+\left(\gamma^{2}-1\right)^{-1} g(x) g^{\mathrm{T}}(x) H_{x}^{\mathrm{T}}(x)\right]+\frac{1}{2}\left[\left(\gamma^{2}-1\right)^{-1}-1\right] \cdot P_{x}(x) g(x) g^{\mathrm{T}}(x) P_{x}^{\mathrm{T}}(x) \\
& \quad+\frac{1}{2} \gamma^{2}\left(\gamma^{2}-1\right)^{-1} H_{x}(x) g(x) g^{\mathrm{T}}(x) H_{x}^{\mathrm{T}}(x)=0, \quad P(0)=0,
\end{aligned}
$$

having the positive definite solution $P(x)=\left(\gamma^{2} /\left(\gamma^{2}-2\right)\right) H(x)$ for $\gamma>\sqrt{2}$. It follows that the controller

$$
\begin{aligned}
& \dot{\hat{x}}=f(\hat{x})-\frac{\gamma^{2}}{\gamma^{2}-2} g(\hat{x}) g^{\mathrm{T}}(\hat{x}) H_{x}^{\mathrm{T}}(\hat{x})+g(\hat{x})\left[y-g^{\mathrm{T}}(\hat{x}) H_{x}^{\mathrm{T}}(\hat{x})\right], \\
& u=-\frac{\gamma^{2}}{\gamma^{2}-2} g^{\mathrm{T}}(\hat{x}) H_{x}^{\mathrm{T}}(\hat{x})
\end{aligned}
$$


robustly stabilizes $\Sigma$ for every perturbation $\Delta$ with $L_{2}$-gain $<1 / \gamma$. By Remark 3.2, $\gamma^{*}$ is given by (26). Since $W=H$ and $V=-H$ we conclude that $\gamma^{*}=\sqrt{2}$, in accordance with the lower bound $\gamma>\sqrt{2}$ as derived above. From a physical point of view, if in (28) $u$ 's denote external forces and $y$ 's are the corresponding (disturbed) generalized velocities, then (30) corresponds to adding damping with regard to the estimated generalized velocities with a damping factor $\gamma^{2}\left(\gamma^{2}-2\right)^{-1}$, tending to $\infty$ for $\gamma \downarrow \gamma^{*}=\sqrt{2}$.

\section{References}

[1] J. Ball, J.W. Helton and M. Walker, $\mathscr{H}_{\infty}$ control for nonlinear systems with output feedback, IEEE Trans. Automat. Control AC-38 (1993) 546-559.

[2] T. Başar and P. Bernhard, $\mathscr{H}_{\infty}$-Optimal Control and Related Minimax Design Problems (Birkhäuser, Basel, 1990 ).

[3] C.A. Desoer and M. Vidyasagar, Feedback Systems: Input-Output Properties (Academic Press, New York, 1975).

[4] J. de Does, Ph.D. Thesis, forthcoming.

[5] J.C. Doyle, K. Glover, P.P. Khargonekar and B.A. Francis, State space solutions to standard $\mathscr{H}_{2}$ and $\mathscr{H}_{\infty}$ control problems, IEEE Trans. Automat. Control AC-34 (1989) 831-846.

[6] K. Glover and D.C. McFarlane, Robust stabilization of normalized coprime factor plant descriptions with $\mathscr{H}_{\circ x}$ bounded uncertainty, IEEE Trans. Automat. Control AC-34 (1989) 821-830.

[7] D. Hill and P. Moylan, The stability of nonlinear dissipative systems, IEEE Trans. Automat. Control AC-21 (1976) 708-711.

[8] D. Hill and P. Moylan, Connections between finite gain and asymptotic stability, IEEE Trans. Automat. Control AC-25 (1980) 931-936.

[9] A. Isidori, $\mathscr{H}_{\infty}$ control via measurement feedback for affine nonlinear systems, Dept. of Systems Science and Mathematics, Washington University, St. Louis, 1992 to appear in: Int. J. Robust Nonlinear Control.

[10] A. Isidori and A. Astolfi, Disturbance attenuation and $\mathscr{H}_{\infty}$ control via measurement feedback in nonlinear systems, IEEE Trans. Automat. Control AC-37 (1992) 1283-1293.

[11] V. Kucera, Algebraic Riccati equation: Hermitian and definite solutions, in: S. Bittanti, A.J. Laub and J.C. Willems, eds., The Riccati Equation (Springer, Berlin, 1991) 53-88.

[12] H. Kwakernaak, Robustness optimization of linear feedback systems, 22nd IEEE CDC, San Antonio, 1983.

[13] H. Kwakernaak, Robust control and $\mathscr{H}_{\infty}$-optimization - tutorial paper, Automatica 29 (1993) 255-273.

[14] W.M. Lu and J.C. Doyle, $\mathscr{H}_{\infty}$-control of nonlinear systems: a class of controllers, Tech. Memo. CIT-CDS93-008, 1993.

[15] D.G. McFarlane and K. Glover, Robust Controller Design using Normalized Coprime Factor Plant Descriptions, LNCIS 138 (Springer, Berlin, 1990).

[16] H. Nijmeijer and A.J. van der Schaft, Nonlinear Dynamical Control Systems (Springer, New York, 1990).

[17] A.D.B. Paice and A.J. van der Schaft, The class of stabilizing nonlinear plant controller pairs, submitted.

[18] J.M.A. Scherpen and A.J. van der Schaft, Normalized coprime factorizations and balancing for unstable nonlinear systems, to appear in: Int. J. Control.

[19] A.J. van der Schaft, On a state space approach to nonlinear $\mathscr{H}_{\infty}$ control, Systems Control Lett. 16 (1991) 1-8.

[20] A.J. van der Schaft, Relations between $\left(\mathscr{H}_{\infty}\right.$-) optimal control of a nonlinear system and its linearization, in: Proc. 30th CDC, Brighton, UK (1991) 1807-1808.

[21] A.J. van der Schaft, $L_{2}$-gain analysis of nonlinear systems and nonlinear state feedback $\mathscr{H}_{\infty}$ control, IEEE. Trans. Automat. Control AC-37 (1992) 770-784.

[22] A.J. van der Schaft, Nonlinear state space $\mathscr{H}_{\infty}$ control theory, H.L. Trentelman and J.C. Willems, eds., Essays on Control: Perspectives in the Theory and its Applications, PSCT14 (Birkhäuser, Basel, 1993).

[23] M. Vidyasagar, Control Systems Synthesis - A Factorization Approach (MIT Press, Cambridge, MA, 1985).

[24] M. Vidyasagar and H. Kimura, Robust controllers for uncertain linear multivariable systems, Automatica (1986) 85--94.

[25] J.C. Willems, Dissipative dynamical systems. Part I: general theory, Arch. Rational Mech. Anal. 45 (1972) 321-351. 